\title{
Adaptive Real Time Eye-Blink Detection System
}

\author{
Mai K. Galab \\ Computer Science \\ Department, Faculty of \\ Computer \& Informatics, \\ Benha University, Benha, \\ Egypt
}

\author{
H.M.Abdalkader \\ Information System \\ Department, Faculty of \\ Computers and Information, \\ Menofia University, Shebien, \\ Egypt
}

\author{
Hala H. Zayed \\ Computer Science \\ Department, Faculty of \\ Computer \& Informatics, \\ Benha University, Benha, \\ Egypt
}

\begin{abstract}
The eye is one of the sense organs that can give users better interaction closer to their need by observing the change of the eyes (open or closed). It is considered as a rich source for gathering information on our daily life. So, it is used in computer science area, especially in human computer interaction. This paper proposes a new system for detecting eye blinks accurately without any restriction on the background and the user does not have to wear any sensors or marks. No manual initialization is required in our proposed system. The proposed system works with the online and offline environment. It automatically classifies the eye as either open or closed at each video frame. The proposed system is tested with the users who wear glasses and the experiments proved its applicability. The proposed system is very easy to configure and use. It is totally non-intrusive and it only requires one low-cost web camera and computer.
\end{abstract}

\section{General Terms}

Computer Vision, Human Computer Interface.

\section{Keywords}

Face Detection, Eye Detection, Blink Detection.

\section{INTRODUCTION}

The eye has a wide range of usage in computer science area, especially in human-computer interaction (HCI) [1]. Eye blinking is one of the informative and non- verbal behavioral cues for solving many problems. Eye blinking has a wide range of applications like the eye typing applications as in [2 5], the detection of driver fatigue as in [6 - 9], determination of concealment-of-intent behaviors, and otherwise. The primary goal of this paper is producing a robust, accurate system to detect eye blinks online and offline for people who wear glasses or not. The proposed system is system without any infrared light, in spite of the most commercial eye tracking systems depend on the infrared (IR) light that give a robust and an accurate result. The main reason for building the proposed system without any infrared (IR) light is be easy to use, robust and non-fatigued or non-intrusive. The use of infrared (IR) light systems gives a robust and very accurate result. But introduces some drawbacks like: first, most users are not able to get a successful calibration result due to false reflections from glasses, interference with the ambient light, or occlusion by eyelids or eyelashes. It would get worse in some situations, such as the users wearing glasses or the ambient light in an outdoor environment. So, most current eye tracking systems can only work in indoor scenario. Second, other users may feel uncomfortable and their eyes tend to dry out as the result of long-time direct exposure to the IR light. Finally, the use of IR source and an IR camera increases the cost such that the prices of current IR-based commercial systems are very expensive [1].
The main purpose of this paper is to develop a real time self adaptation eye blink system. The developed system is provided to be a low robust than similar systems.

The proposed system can be used in many computer vision applications such as eye typing, driver blink rate monitoring, and lie detection by measuring blink detection patterns, and eye-gaze input for human computer interaction, etc.

In the Commercial field, devices that require the users to wear no equipment on their heads cost in excess of $\$ 35,000$. The goal of this research is to develop and evaluate a system that is both low cost and also does not require the user to wear any equipment. The selected way of doing this in the proposed system is to use only the Laptop Webcam. Comparing the proposed approach with the state-of-art eye tracking methods based on IR-light shows that our proposed system is very simple and very low cost system. The proposed system can detect closing and opening for eye at each video frame separately.

This paper is organized as follows: Section 2 covers the related work. Section 3 describes the proposed method. Experimental results are shown in Section 4. Finally, Section 5 presents the conclusion.

\section{RELATED WORK}

In this section we try to cover all methods that work with eyemovement, especially with eye blink systems and its applications.

In previous research on vision-based blink detection, as in [10 -13] the blink detection system built on finding the difference between successive images (that means the result of the current image depends on the previous one). The limitation of the previous methods is that head movement is not allowed, which is a serious disadvantage for actual blink-based applications. To overcome this limitation, many studies have proposed.

Morris et al. [14] proposed the eye blink detection method using spatio-temporal filtering and Lucas-Kanade feature tracking to locate the position of the head and to extract eyelid movements, they report good blink detection result (95\% true position), but head movements affect the variance map computation and cause a sharp drop in performance. The primary contribution of this method is the automatically initializing the eyes' locations in an image sequence for real time eye tracking applications.

Sirohey et al. [15] proposed an approach for determining eye blinks by locating eye corners, eyelids and irises in each frame of an image sequence, and analyzing their movements to determine changes in gaze direction and blinking, 
respectively. Motion information is estimated using normal flow. Determining the head-independent motions of the irises and eyelids by stabilizing for the head motion. The authors claim their algorithm can track iris and eyelid motion more that $90 \%$ of the time, but not in real time.

Grauman et al. [16] proposed two real-time systems (Blink Link and EyebrowClicker) that consistently run at frame rate, are completely non-intrusive, and require no manual initialization, or special lighting. The "BlinkLink" system can reliably classify blinks as voluntary or involuntary based on their duration. "EyebrowClicker" can reliably interpret the motion of the eyebrows and detect a raised eyebrow based on its distance to the eye and the duration of the motion. The two video-based interaction systems were tested and the results demonstrate overall detection accuracy of $95.6 \%$ for "BlinkLink" and $89.0 \%$ for "EyebrowClicker". In both the blink and eyebrow raise systems, Large and sudden changes from moving the camera or a light source mid-use will influence the system. The system is very sensitive to specularity. So, it's not giving good results with the users who wear glasses.

Chau et al. [17] proposed a blink detection system based on real time eye tracking with USB camera using the template matching for detecting eyes and analyzing blinks. They report $95 \%$ overall blink detection accuracy. The main benefit of such template-based approach is its low computational complexity. The downside is that it only distinguishes between two eye states, open and closed. Any movement inbetween is not well defined.

Recently, Heishman et al. [18] proposed a method for using image flow analysis to compute problematic eye blink parameters. The flow analysis produces the magnitude and direction of the eyelid movement. A deterministic finite state machine uses the eyelid movement data to compute blink parameters (e.g., blink count, blink rate, and other transitional statistics) for use in human computer interaction applications across a wide range of disciplines.

In another study, Pan et al. [19] used a boosted classifier modelled by a Hidden Markov Model (HMM) to detect the eye-blink rate. Their method operates in real-time on $320 \times$ 240 webcam images, detecting more than $96 \%$ of eye blinks. The method should face difficulties when non-standard eye motion occurs, such as partial blinks.

Orozco et al. [20] proposed method using two appearancesbased trackers: the first one tracks iris movements while the second one focuses on eyelids and blinking. Using low resolution input video and a simple appearance model, the method reportedly runs in real-time, achieving good tracking results. Authors didn't try to detect eye blinks; however the method could be used for this purpose.

Bacivarov et al. [21] proposed a straightforward proof-ofconcept model, having as an advantage that it models properly the eye region for both eyes open and closed by using A statistical Active Appearance (AAM) based techniques. It can be adapted to work in real-time. An eye tracker and blink detector were also developed using this model.

Divijak et al. [22] proposed a method that works with a recorded video taken by web camera processes the frames and detects hazardous behaviour based on user's eye dynamics and blink patterns. His system builds on template-based method. The experimental results show that system capable of detecting common cases of fatigue behaviour with performance more than $95 \%$. The system has some problems with false detections in cases where the eyes are rapidly moving up and down.

Lee et al. [23] developed a new method for blink detection, which maintains its accuracy even if the facial pose changes, which depends on four steps. First, the face and eye regions are detected by using both the Ada Boost face detector and the Lucas-Kanade-Tomasi (LKT) based method in order to be robust to the facial pose. Secondly, the eye "open" and "closed" states are determined on the basis of two features: the ratio of height to width of the eye region in a still image, and the cumulative difference in the number of black pixels seen in the eye region using an adaptive threshold in successive images. Thirdly, the accuracy of determining the eye state when it is open or closed is increased by combining the above two features on the basis of the Support Vector Machine (SVM). Finally, the SVM classifier for determining the eye state is adaptively selected according to the facial rotation. The performance of eye-blink detection of this method up to $96 \%$.

Naveed et al. [24] presented an efficient eye tracking system having a feature of eye blink detection for controlling an interface. His system is able to track eye movements efficiently and accurately by using the pupil portion and can accurately detect eye blinks whether voluntary and involuntary. The system can track eye portion with the $90 \%$ detection accuracy. The system is expanded to work in real time using recorded videos.

\section{THE PROPOSED SYSTEM}

Applications based on eye-blink detection have increased because it is suitable for people who are either normal or disabled (that only can blink their eyes). Determining eye status (i.e. Open or closed) is more difficult than just determining eye locations, because of their small region occupancies on the face information or weak contrast between the eye and the surrounding skin [25]. The proposed system is a new system for detecting eye blink feature from a video. It was tested for online and offline video. It does not require any dedicated and expensive hardware. A webcam is the only device required. The system output detects the information about the eye states either it is open eye or closed, which can be applied in many applications like: eye typing, detection of driver fatigue and etc. This section introduces the architecture of the proposed system and describes the principle of each component in more details.

\subsection{System Architecture}

The proposed system can be divided into five stages: capture image from live video or recorded video, face detection, eye detection, image enhancement, and blink detection result. The first stage is face detection. The second stage is eye detection. The used eye region is determined at the third stage; after marking the face region, the left eye and the right eye regions by rectangles in the used face. For quickly and accurately detect eye state we apply the enhancement stage on the extracted eye region at the fourth stage. Finally the eye states open and closed are generated at the final stage. Figure 1 shows the overview of the system architecture. 


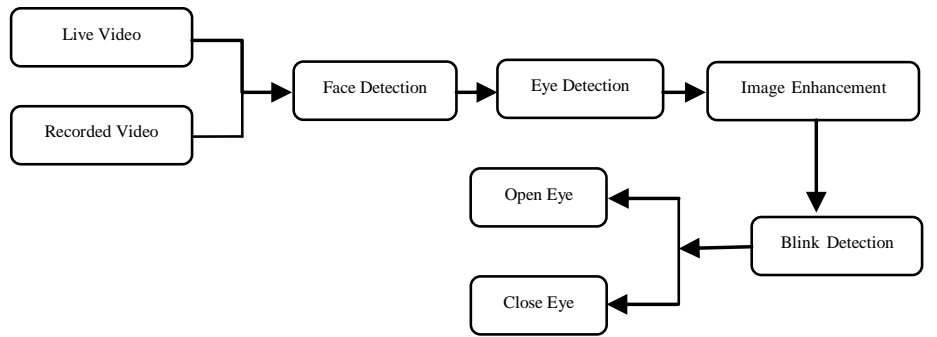

Fig 1: System block diagram

The details of each component will be described in the following subsections.

\subsubsection{Image Capture:}

As the system works in, either online or offline stages, the capture stage have two phases. In online phase, the video is captured and recorded, and then whole video is extracted into frames. On the other hand, the online phase is working by capture an instant image and decide directly if it is open or closed.

\subsubsection{Face Detection:}

Face detection is an indispensable step and it is the actual first step of the system framework. It determines if the later stages are going to run or not. In the proposed system Viola Jones algorithm [26] is applied for face detection and tracking as shown in Figure 2. The Viola Jones algorithm is more efficient for tracking than the AdaBoost Algorithm when working with multiple image frames. Viola Jones can detect more than face if the image contains multi faces (it can detect the correct face with the existing of other people or objects). It can track different types of facial views, not only the frontal view like AdaBoost that needs to a Lucas-Kanade-Tomasi (LKT) based method to support non-frontal faces [23]. Viola Jones is characterized by being extremely fast and achieving high detection rates. The basic idea of this algorithm is to slide a window across the image and evaluate a face model at every location, this window or bounding box serves to restrict the region of the image that is searched for the eyes.
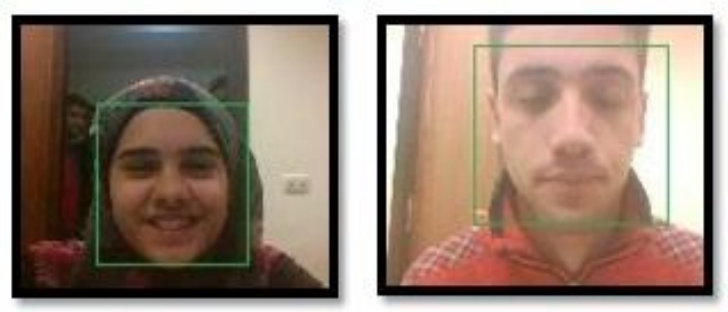

Fig 2: Face detection results after using viola Jones Algorithm

\subsubsection{Eye Detection}
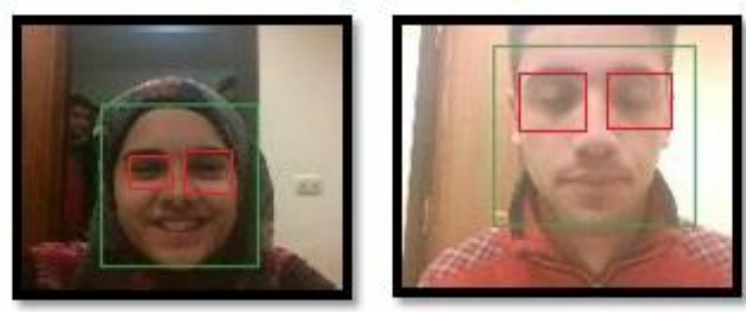

Fig 3: Localized eyes from face image: Eye detection results after using viola Jones Algorithm

The proposed system will focus only on eye region. So, the needed step is extracting the eye region from whole face. The eye region is extracted from the human face by discarding the lips, nose and other unwanted regions of the face as shown in Figure 4.
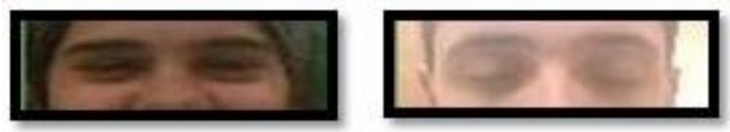

Fig 4: Extract eye part

The proposed system is started by extracting the eye region. But, with the use of the eye region that consists of the eyebrows, the eyelid, eyelashes, pupil, and iris appeared some mistakenly results in the classification step that some closed eyes are classified as open ones. So, the decision was discarding the eyebrows from eye region. By simply dividing the eye region into three horizontal parts and extracting the first horizontal part of this eye region to segment the eyebrows, as shown in Figure 5. Removing the eyebrows because the existence of this part makes conflict in results.
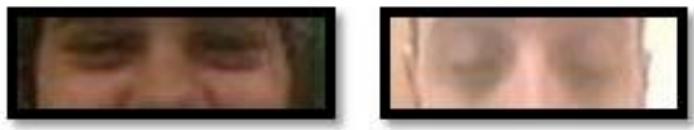

Fig 5: Final eye part after extracting eyebrows

Using eye region as in Figure 5 improves the result and decreases error rate.

\subsubsection{Image Enhancement \\ 3.1.4.1 Illumination-Normalization and \\ Binarization of The Detected Eye Region:}

The process of determining the eye state (open or closed) firstly needs to binarized image, but the most significant problem that facing image binarization is the effect of lighting variations in imagery. The variation in the lighting increases the noise rate in image. Figure 6 shows an example of a binarized eye image having an illuminative variation. As shown in Figure 6 (a) the threshold determined by 40 right eye disappeared and the left eye is clearly displayed. By increasing the threshold to 55 to enhance the visibility of the left eye the right eye will distort as shown in Figure 6 (b).

(a)

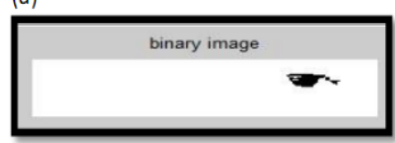

Fig 6: Binarized images

(b)

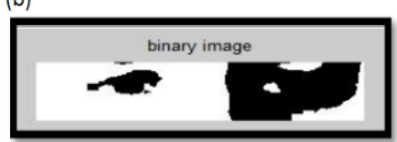

So, to solve this problem illumination-normalization was applied on the detected eye region by using a Lee algorithm in [23] which converts the RGB input image to an $L * a * b *$ image (L). An illuminative component is acquired by applying a $31 \times 31$ median filter to the L image, as shown in Figure 7 (b). Then invert the illuminative image that was obtained, as shown in Figure 7 (c). After that add the inverted image to the original L image, as shown in Figure 7 (d). Using this algorithm in the proposed system for the illumination process specially, because it is fast and not time-consuming. 

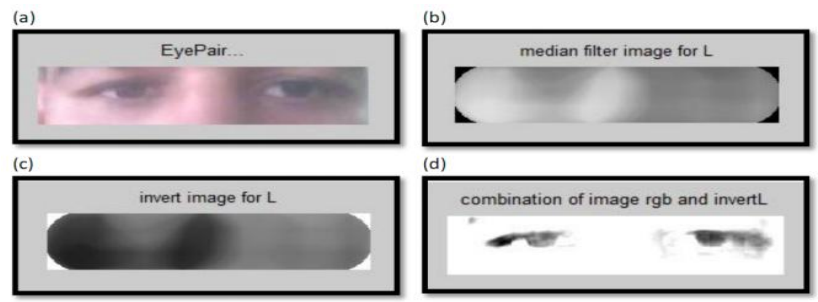

Fig 7 :The image obtained by applying illumination process

After illumination normalization we binarized eye image by applying Otsu threshold on the resulting image of Figure 7 (d) as shown in Figure 8. After trying and testing other kinds of thresholding, the Otsu threshold was preferred in this method because the local thresholding or local adaptive binarization methods not effective with these kinds of image which have a low resolution.

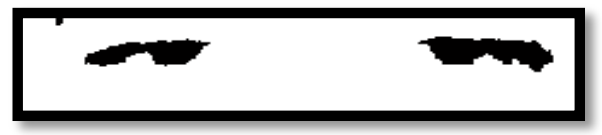

Fig 8: Binarized images by Otsu threshold

\subsubsection{The Morphological Operation on the \\ Detected Eye Region:}

After binarizing the eye region determining the eye state (closed or open) is still a challenge problem because some open eyes are classified as closed ones. So, to overcome this problem, some processes are applied to the binary image before the step of classification starts by smoothing the image by applying an average filter on binary images, as shown in Figure 9 (a) then applying dilation and erosion, filters, respectively, as shown in Figure 9 (b). Finally Canny edge detector is applied to the resulted image to detect exactly the two eyes as in Figure 9 (c) and in Figure 9 (d).
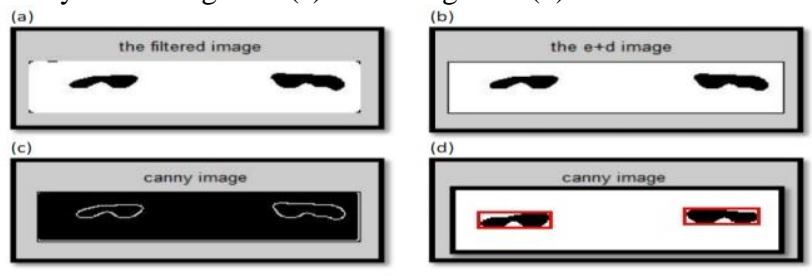

Fig 9: Image after morphological operations

\subsubsection{Blink Detection}

The blink detection stage is the final stage in our proposed system. The blink detection process divided into two parts to decide if the eye is closed or open. The two parts described in a more detailed blow:

\subsubsection{The Difference between the Number of Black Pixels in Two Parts of Eye Region:}

This step used to determine if the eye is open or closed by receiving two canny eye objects from the image enhancement stage, the first eye object refers to the left eye, and the second refers to the right eye as shown in Figure 9 (d). The proposed system works separately on each object, that means the proposed system repeat the same process on each eye object by dividing each eye object (left and right) horizontally into two equal parts. For each eye object finding the difference between the number of black pixels in the bottom part of the eye object to the number of black pixels in the above part, and if the difference is greater than zero this means that the eye is closed otherwise the eye is open, as shown in Figure 10.

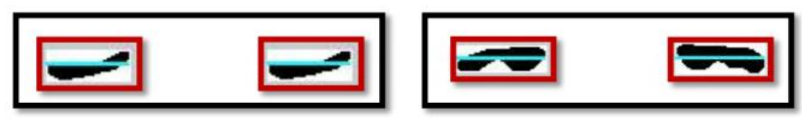

Fig 10: Eye object divided horizontally into two parts

\subsubsection{Compute Ratio of Black Pixels to White} Pixels in the Above Part:

To enhance the accuracy of determination of an opened or closed eye, this step was added as a supplementary step to the proposed system. Through testing the proposed system some images with special case are found, these images are open images, but with difference greater than zero. So, the proposed system will classify these images as closed images to avoid this mistake the proposed system was supplied by this extra step that tests the images with difference greater than zero if they are open or closed. At this step, the proposed is concentrated on the upper part of eye object only. Then compute the ratio of black pixels to white pixels in the above part of the eye as shown in Figure 11, if the number of black pixels is greater than that of white pixels this means that the eye is opened otherwise it is closed.

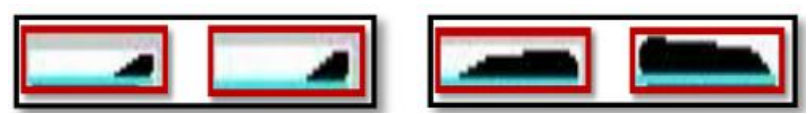

Fig 11: Example of the above part of close and open eye

\section{EXPERIMENTAL RESULTS}

The implementation of the proposed system faced several areas of difficulty before achieving the following advantages:

1. Identifying face and eye location.

2. Detecting blinks of the eyes with glasses or without.

3. Being able to detect the information of eye (open or closed) in the real-time environment.

Performance evaluation is very crucial and tedious task and at the end of the development process it is significant to show that the final proposed system achieves an acceptable level of performance and that it represents a significant improvement over existing systems or not. So, we test our proposed system with two environments: the offline and the online.

The System is developed by a MATLAB R2012a.This implementation is running on Windows 732 bit operating system with an Intel® core2TM Duo CPU $(2.20 \mathrm{GHz})$ and 3GB of RAM. The camera in the proposed system is HP WebCam101 that built in camera embedded in the used laptop. Webcam with video size equal $640 \mathrm{x} 480$. For evaluating our proposed system we select the two measurements are usually used in the evaluation process "Recall" and "Precision". Recall: is the average detection rate, which is calculated by the equation (1):

$$
\text { Recall }=\frac{\mathrm{TP}}{\mathrm{TP}+\mathrm{FN}}
$$

Where TP is the number of true positives (implies correct detection of eye-state means open eye returned as open eye) and $\mathrm{FN}$ is the number of false negative (closed eye returned as open eye).

Precision: is calculated by the equation (2):

$$
\text { Precision }=\frac{\mathrm{TP}}{\mathrm{TP}+\mathrm{FP}}
$$


Where TP is the number of true positives (implies correct detection of eye-state) and FP is the number of false positives (implies an open eye returned as closed eye).

In order to evaluate the performance of the proposed system several experiments have been designed. These experiments are divided into two sets: Offline tests, and Real time tests.

\subsection{The Offline Tests}

The proposed system was tested on people with glasses or without glasses. First, we start the testing process with people without glasses. Second, for testing process with people who is wearing glasses.

\subsubsection{The testing process with people without glasses:}

In the testing process five different databases for people who not wearing glasses are used. The first two databases are our own database, which are captured from laptop webcam and the spatial resolution is $640 \times 480$ pixels. These two databases are for different people. Their ages are between 25 and 35 . The number of frames in the first video is 120 frames. The number of frames in the second video is 192 frames. The third database is the Talking Face video database ( http://wwwprima.inrialpes.fr/FGnet/data/01TalkingFace/talking_face.htm 1.Last Visit: July, 2014) which contains one video having 999 frames captured from one Caucasian male whose age is estimated as 20s or 30s. It includes 61 blinks in all. The fourth database is self-collected dataset, for female with age around 25 years, which contains video having 149 frames. The fifth database is the ZJU Eye blink Database (http://www.cs.zju.edu.cn/ gpan/database/db_blink.html Last Visit: July, 2014) which comprises 20 short videos having 2950 frames $(320 \times 240$ pixels $)$ captured from 20 Chinese people (13 males and 7 females, dark brown eye colors, ages are estimated as 20s) at a capturing speed of 30 frames/s. They include 255 blinks at all. Examples of databases that used in the proposed system are shown in Figure 12.

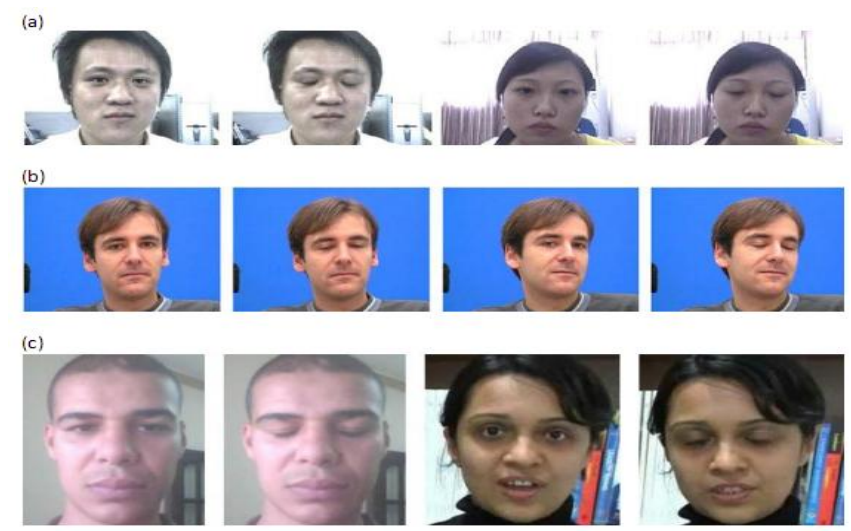

Fig 12: Examples of detecting eye blink on different data sets. (a) Some cases from used database in the ZJU

Database. (b) Talking Database. (c) Our own Database.

This part begins by testing the accuracy of the proposed system to know the total accuracy that achieved by the proposed system. The result is shown in Figure 13 based on the equation (3):

Accurcy $=\frac{\text { Number of correcting cases }}{\text { corrected }+ \text { incorrected }}$

\section{Accurcy of Our Method With Different Datasets without Glasses}

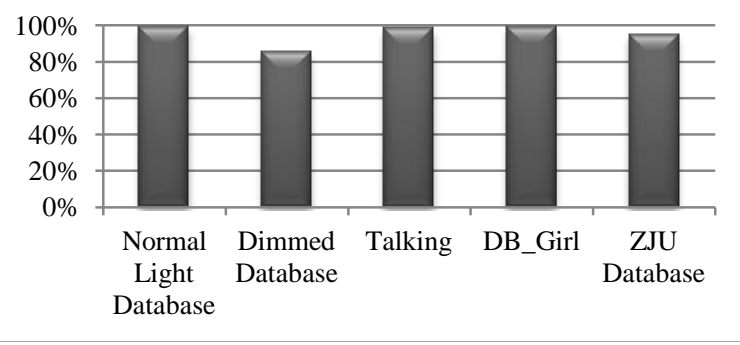

Fig 13: Accuracy of The Proposed Method

This experiment demonstrates the performance of the proposed system under different illumination conditions. The Table I lists the result of "Recall" and "Precision" that obtained using the different databases: ZJU, Talking, and our own databases. The results are varied from dataset to another as a result of the resolution of the camera and the strength of the ambient lighting. In the dataset "Our Own Database1" the web cam with low resolution was used. The result of the recall was $94 \%$ and the result of precision was $100 \%$. In the dataset "Our Own Database2" the web cam with low resolution was used. In this dataset the ambient lighting decreased so, the images became darker than the first dataset "Our Own Database1", and the result of recall became $66 \%$ and the result of precision became 99\%. With the dataset "DB_Girl" give result to recall was $100 \%$ and with precision $99 \%$ because the resolution of cam better than our web cam and the lighting conditions more batter. The result of recall and precision are 96\% and 96\%, respectively, with "Talking" data set. The result of recall and precision are $95 \%$ and $98 \%$, respectively, with "ZJU Database" data set. The proposed system achieved an average Recall of $90.5 \%$ and Precision of $98 \%$.

Table 1 .The Recall and Precision results of the blink detection

\begin{tabular}{|c|c|c|}
\hline Database & Recall & Precision \\
\hline Our Own Database1 & $94 \%$ & $100 \%$ \\
\hline Our Own Database2 & $66 \%$ & $99 \%$ \\
\hline Talking & $96 \%$ & $96 \%$ \\
\hline DB_Girl & $100 \%$ & $99 \%$ \\
\hline ZJU Database & $95 \%$ & $98 \%$ \\
\hline Average & $90.5 \%$ & $98 \%$ \\
\hline
\end{tabular}

The proposed system results were compared with the result of the method that used by [23]. The result of comparing recall and precision in our method with the method that used by [23] on the standard databases ZJU and Talking are respectively shown in Fig14 and Figure 15. After the comparison the proposed system have a higher results with " recall " and " precision " more than that appeared in the method in Ref [23]. 


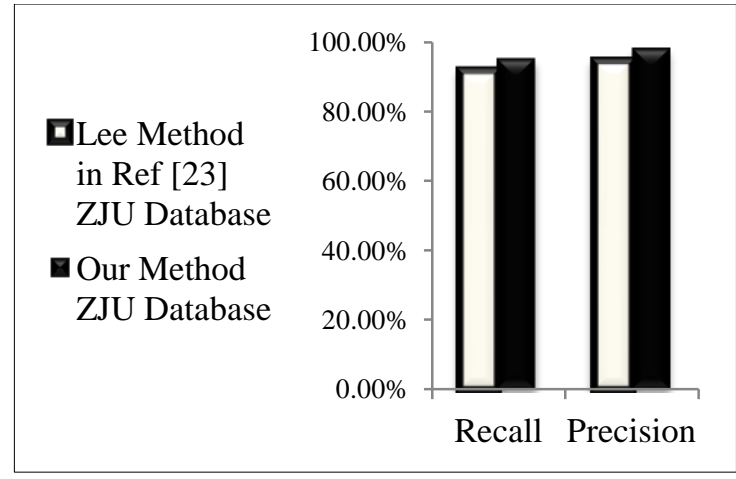

Fig 14: Comparison recall and precision results between our method and method in ref [23] on ZJU dataset

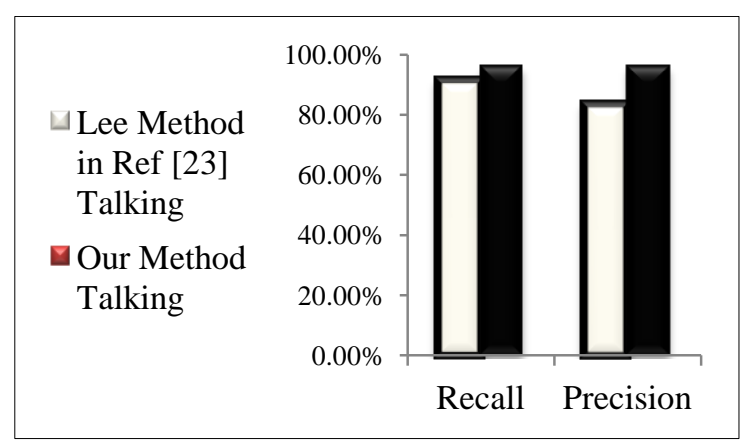

Fig 15: Comparison recall and precision results between our method and method in ref [23] on Talking Data Set.

Unfortunately, we cannot compare those results of our proposed system with other published work, because there is no standard evaluation metrics used in all research.

\subsubsection{The testing process with people with glasses:}

In the testing process four different databases are used. The first two databases are our own database, which are captured from laptop webcam and the spatial resolution is $640 \times 480$ pixels. These two databases for different people their ages between 25 and 35 . The number of frames in the first video is 60 frames. The number of frames in the second video is 41 frames. The third database is the ZJU Eye blink Database (http://www.cs.zju.edu.cn/ gpan/database/db_blink.html Last Visit: July, 2014) which comprises 20 short videos having 2950 frames $(320 \times 240$ pixels $)$ captured from 20 Chinese people (13 males and 7 females, dark brown eye colors, ages are estimated as 20s) at a capturing speed of 30 frames/s. They include 255 blinks at all. Examples of databases that are used in the proposed system are shown in Figure 16. The fourth database is the BioID face database (http://www.bioid.com/index.php?q=downloads/software/bioi d-face-database.html . Last Visit: July, 2014). We test about 480 images from this database with the proposed system and this number is all images which contain people with glasses in the whole database. Samples from BioID face database are shown in Figure 17.
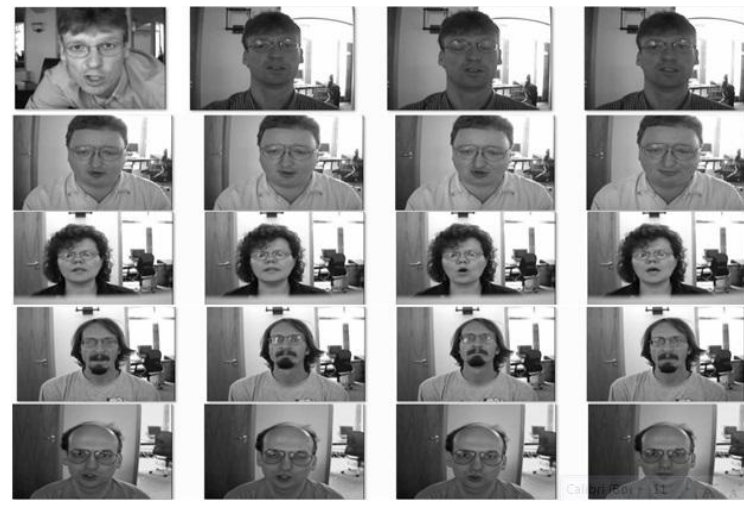

Fig 16: Examples of detecting eye blink on different data sets. (a), (b), (c) are some cases from used database in the ZJU Database. (d) Our own Database.

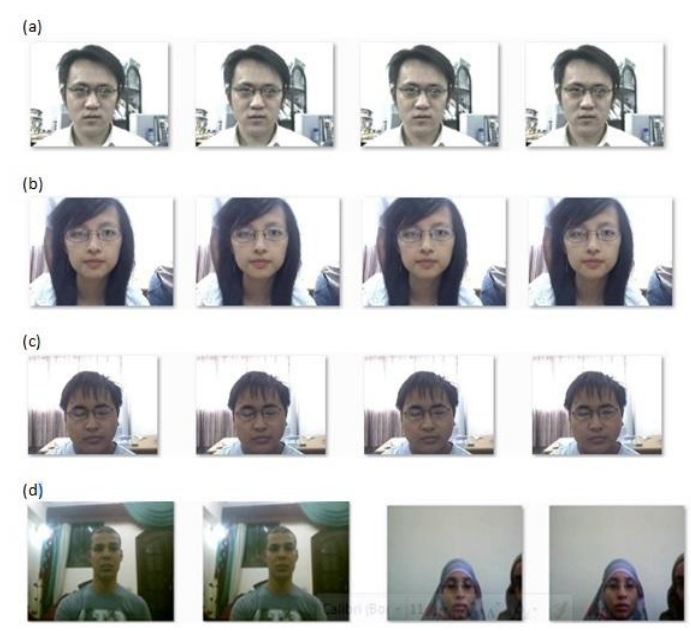

Fig 17: Samples from BioID face dataset

In order to measure the accuracy of the eye blink detection system, we used the equation (3) and the results are shown in Figure 18.

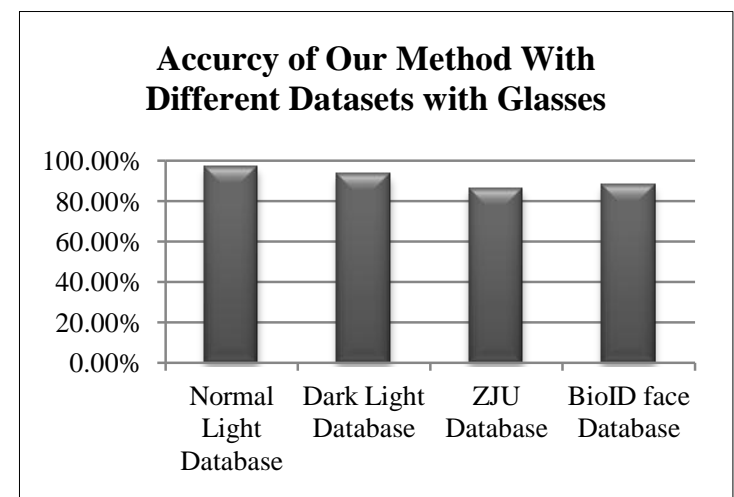

Fig 18: The accuracy of the proposed system with different data sets.

A specularity occurs where the light source rays intersect with certain surfaces, such as a user's eye glasses and this fact cause mislead some blink systems. Although, the glasses cause a specularity and the eye appearance changes with glasses, the proposed system proved its applicability to work with glasses. Table 2 shows the result of applying the proposed system to people with glasses. We can see that the proposed system can still detect eye state (open or closed) 
accurately for people with glasses. The proposed system achieved an average Recall of $93.1 \%$ and Precision of $95 \%$.

Table 2. The Recall and Precision results of the proposed system with different data sets

\begin{tabular}{|c|c|c|}
\hline Database & Recall & Precision \\
\hline Our Own Database1 & $93 \%$ & $93 \%$ \\
\hline Our Own Database2 & $96.7 \%$ & $99 \%$ \\
\hline ZJU Database & $89.3 \%$ & $93 \%$ \\
\hline BioID face Database & $100 \%$ & $90 \%$ \\
\hline Average & $93.1 \%$ & $95 \%$ \\
\hline
\end{tabular}

\subsection{The Real Time Tests}

In real time testing two different types of light sources were used in the data set: sunlight from a window and lamplight. The proposed system can work accurately and detect eye state (open or closed) in an online environment.

The proposed system was tested with different people their ages between 20 and 35 . The number of frames in this test more than 1000 frames. The proposed system achieved an average Recall of $97.2 \%$ and Precision of $94.4 \%$.

Table 3 shows the result of Recall and Precison of the database that used sunlight in the window and the level of light with this dataset is stronger than the light that comes from the lamp.

Table 4 shows the result of Recall and Precison of the database that used lamplight and used different kinds of people. Video_7 refers to a female wears a scarf, and no closer to our web cam, and result of Recall is $96 \%$ and Precision is $94 \%$. Video 8 refers to a female wears a scarf, but more closer to our web cam than Video_7, and result of Recall is $96.7 \%$ and Precision is 96.7\%. Video_9 refers to not a veiled female with black hair, and result of Recall is $96.9 \%$ and Precision is 98\%. Video_10 refers to a male with colored eyes (green eyes), and result of Recall is $95 \%$ and Precision is 92\%. Video_11 refers to a male very close from our web cam, and result of Recall is $99 \%$ and Precision is $99 \%$. Video_12 refers to a male with small eyes like Chinese eyes, and result of Recall is $92 \%$ and Precision is $90 \%$.

Table 3. The Result of Recall and Precision with the first kind of database. With test time is 1 frame per second.

\begin{tabular}{|c|c|c|c|c|}
\hline Datasets & Test Time & $\begin{array}{c}\text { Number of } \\
\text { Frames }\end{array}$ & Recall & Precision \\
\hline Video_1 & $\begin{array}{c}\text { 1 Frame per } \\
\text { Second }\end{array}$ & 25 Frame & $100 \%$ & $92 \%$ \\
\hline Video_2 & $\begin{array}{c}\text { 1 Frame per } \\
\text { Second }\end{array}$ & 50 Frame & $100 \%$ & $94 \%$ \\
\hline Video_3 & 1 Frame per & 75 Frame & $100 \%$ & $97 \%$ \\
\hline
\end{tabular}

\begin{tabular}{|c|c|c|c|c|}
\hline & Second & & \\
\hline Video_4 & $\begin{array}{c}\text { 1 Frame per } \\
\text { Second }\end{array}$ & 100 Frame & $100 \%$ & $97 \%$ \\
\hline Video_5 & $\begin{array}{c}\text { 1 Frame per } \\
\text { Second }\end{array}$ & 150 Frame & $96.9 \%$ & $88.2 \%$ \\
\hline Video_6 & $\begin{array}{c}\text { 1 Frame per } \\
\text { Second }\end{array}$ & 200 Frame & $95 \%$ & $95 \%$ \\
\hline
\end{tabular}

Table 4. The Result of Recall and Precision with the first kind of database. With test time is 10 frames per second.

\begin{tabular}{|c|c|c|c|c|}
\hline Datasets & Test Time & $\begin{array}{c}\text { Number of } \\
\text { Frames }\end{array}$ & Recall & Precision \\
\hline Video_7 & $\begin{array}{c}\text { 10 Frames } \\
\text { per Second }\end{array}$ & 141 Frame & $96 \%$ & $94 \%$ \\
\hline Video_8 & $\begin{array}{c}\text { 10 Frames } \\
\text { per Second }\end{array}$ & 122 Frame & $96.7 \%$ & $96.7 \%$ \\
\hline Video_9 & $\begin{array}{c}\text { 10 Frames } \\
\text { per Second }\end{array}$ & 170 Frame & $96.9 \%$ & $98 \%$ \\
\hline Video_10 & $\begin{array}{c}\text { 10 Frames } \\
\text { per Second }\end{array}$ & 200 Frame & $95 \%$ & $92 \%$ \\
\hline Video_11 & $\begin{array}{c}\text { 10 Frames } \\
\text { per Second }\end{array}$ & 150 Frame & $99 \%$ & $99 \%$ \\
\hline Video_12 & $\begin{array}{c}\text { 10 Frames } \\
\text { per Second }\end{array}$ & 98 Frame & $92 \%$ & $90 \%$ \\
\hline
\end{tabular}

\section{CONCLUSIONS}

Low cost systems are not commonly used on the market. Most of the eye blink systems limited on the infrared light systems that work with special hardware and give very accurate results, but with a high cost. So, the main goal of our proposed system is trying to solve this problem. The proposed system starts by detecting human face. Then the eye region is extracted from the detected face. Finally eye blink is detected using shape measurement and other different steps are used to determine eye state. The proposed system can work under different illumination conditions. The Experimental results show that this new system achieves a satisfied performance for eye blink detection. The final results show that the system accuracy is approximately $98 \%$ with offline tests and $95 \%$ with online tests.

\section{REFERENCES}

[1] C. Chou, W. Peng and Y. Hung. 2008. Real-Time Three-Stage Eye Feature Extraction. Technical Report, National Taiwan University, Taipei, Taiwan.

[2] B. Ashtiani and I.S. MacKenzie. 2010, BlinkWrite2 : an improved text entry method using eye blinks. In Proceedings of the 2010 Symposiumon Eye-Tracking 
Research \& Applications, ETRA'10 ,pp.339345,NewYork, NY, USA, ACM.

[3] M.Barrett, H.Skovsgaard, and J.SanAgustin. 2009. Performance evaluation of a Low-Cost gaze tracker for eye typing. In Proceedings of the $5^{\text {th }}$ Conference on Communication by Gaze Interaction, COGAIN'09, pp.13-17.

[4] M.Porta, A.Ravarelli, and G.Spagnoli. 2010. ceCursor, a contextual eye cursor for general pointing in windows environments. In Proceedings of the 2010 Symposiumon Eye-Tracking Research \& Applications, ETRA '10,pp.331-337, NewYork, NY, USA.

[5] O.Tuisku, P.Majaranta, P.Isokoski, and K.Räihä. 2008. Now dasher! dashaway! : longitudinal study of fast text entry by eye gaze. In Proceedings of the 2008 Symposiumon Eye-Tracking Research \& Applications, ETRA'08, pp.19-26, NewYork, NY, USA. ACM. ACMID: 1344476.

[6] M. Sugur, B. Hemadri and P.Kulkarni. 2013. Drowsiness Detection Based On Eye Shape Measurement In International Journal of Computer and Electronics Research, vol 2, No 2.

[7] M. Singh, G. Kaur. 2012 .Drowsy Detection On Eye Blink Duration Using Algorithm. In International Journal of Emerging Technology and Advanced Engineering,vol 2, No 4 .

[8] V. Hal, Bryan,.2013. Real-time Stage 1 Sleep Detection and Warning System Using a Low-Cost EEG Headset. Master Thesis, Grand Valley State University.

[9] A. Sahayadhas, K. Sundaraj and M. Murugappan. 2012. Detecting Driver Drowsiness Based on Sensors: A Review. In Sensors — Open Access Journal. pp. 1693716953.

[10] P. BalaL, K. Talmi, and J. Liu. 1997. Automatic detection and tracking of faces and facial features in Video sequences. In Proceedings of picture coding symposium, .pp. 251-256.

[11] J. Crowley, and F. Berard. 1997. Multimodal tracking of faces for video communications. In Proceedings of international conference on CVPR, .pp. 640-645.

[12] K. Grauman, M. Betke, J. Gips, and GR. Bradski. 2001. Communication via eye blinks-detection and duration analysis in real time. In: Proceedings of the international conference on CVPR; .pp. 1010-1017.

[13] S. Kawato, and N. Tetsutani. 2002. Detection and tracking of eyes to gaze-camera control. In Proceedings of international conference on vision interface; .pp. 348455 .
[14] T. Morris, P. Blenkhorn, and F. Zaidi. 2002. Blink Detection for Real-Time Eye Tracking. Journal of Network and Computer Applications, Vol.25, No.22, .pp. 129-143.

[15] S. Sirohey, A. Rosenfeld, and Z. Duric. 2002. A Method of Detecting and Tracking Irises and Eyelids in Video. Pattern recognition, Vol. 35 NUM 6, .pp. 1389-1401.

[16] K. Grauman , M. Betke, J. Lombardi, J. Gips, G. R. Bradski. 2003. Communication Via Eye Blinks and Eyebrow Raises: Video-Based Human-Computer Interfaces. Springer-Verlag, .pp. 359-373.

[17] M. Chau and M. Betke. 2005. Real Time Eye Tracking and Blink Detection With USB Cameras. Technical Report, Boston University Computer Science.

[18] R. Heishman, and Z. Doric. 2007. Using Image Flow to Detect Eye Blinks in Color Video. In Proceedings of the IEEE Workshop on Applications of Computer Vision, .pp. 52.

[19] G. Pan, L. Sun, Z.Wu, and S. Lao. 2007. Eye BlinkBased Anti-Spoofing in Face Recognition from A Generic Web Camera. In Proceedings of the 11th IEEE international conference on computer vision, pp. 1-8.

[20] J. Orozco, F. Xavier Roca, and J. Gonzàlez. 2009. RealTime Gaze Tracking with Appearance-Based Models. Journal of Machine Vision and Applications,Vol.20, pp. 353-364.

[21] I. Bacivarov, M. Ionita, and P. Corcoran. 2008. Statistical Models of Appearance for Eye Tracking and Eye-Blink Detection and Measurement. IEEE Transactions on Consumer Electronics, Vol.54, No.3, pp. 1312-1320.

[22] M. Divijak, and H. Bischef. 2009. Eye Blink Based Fatigue Detection for Prevention of Computer Vision Syndrome. In Proceedings of the IAPR conference on machine vision Applications, pp. 10-14.

[23] W. Lee, E. Lee, and K. Park. 2010. Blink Detection Robust to Various Facial Poses. Journal of Neuroscience Methods , pp. 356-372.

[24] S. Naveed, B. Sikander, and M. Khiya. 2012. Eye Tracking System with Blink Detection. Journal of Computing, Vol.4, No.3.

[25] P. Corcoran, J. Bacivarov, and M. C. Jonita. 2008. A Statistical Modelling Based System for Blink Detection in Digital Cameras. In Proceedings of the IEEE, .pp. 1-2.

[26] P. Viola and M.J. Jones. 2004. Robust real-time face detection. International Journal of Computer Vision, vol. 57 , no. 2, 137-154 